EPJ Web of Conferences 43, 04003 (2013)

DOI: $10.1051 /$ epjconf/20134304003

(C) Owned by the authors, published by EDP Sciences, 2013

\title{
X-ray emission from hot subdwarfs with compact companions
}

\author{
S. Mereghetti ${ }^{1, a}$, N. La Palombara ${ }^{1}$, P. Esposito ${ }^{1}$ and A. Tiengo ${ }^{1,2}$ \\ ${ }^{1}$ INAF - IASF Milano, via Bassini 15, 20133 Milano, Italy \\ 2 IUSS, piazza della Vittoria 15, 27100 Pavia, Italy
}

\begin{abstract}
We review the X-ray observations of hot subdwarf stars. While no X-ray emission has been detected yet from binaries containing B-type subdwarfs, interesting results have been obtained in the case of the two luminous O-type subdwarfs HD 49798 and $\mathrm{BD}+37^{\circ} 442$. Both of them are members of binary systems in which the X-ray luminosity is powered by accretion onto a compact object: a rapidly spinning (13.2 s) and massive $\left(1.28 M_{\odot}\right)$ white dwarf in the case of HD 49798 and most likely a neutron star, spinning at $19.2 \mathrm{~s}$, in the case of $\mathrm{BD}+37^{\circ} 442$. Their study can shed light on the poorly known processes taking place during common envelope evolutionary phases and on the properties of wind mass loss from hot subdwarfs.
\end{abstract}

\section{INTRODUCTION}

Hot subdwarfs are evolved low-mass stars that lost most of their hydrogen envelopes and are now in the stage of helium core burning. A possible mechanism responsible for the loss of the massive $\mathrm{H}$ envelopes necessary to form hot subdwarfs is mass transfer in a binary. Indeed, many hot subdwarfs are members of binary systems $[18,22]$, supporting the idea that non-conservative mass transfer played a role in the formation of these stars. Evolutionary models predict that the most of the subdwarf companions in binaries with orbital period shorter than $\sim 10$ days should fall into two main groups: late type main sequence stars and white dwarfs [9].

X-rays can be used to identify hot subdwarfs with compact companions: they can originate from surface thermal emission of neutron stars or sufficiently hot white dwarfs or can be produced if the compact object accretes mass from the subdwarf at a sufficiently high rate. This is well illustrated by our recent results on the sdO binaries discussed below: HD 49798 and $\mathrm{BD}+37^{\circ} 442$. Furthermore, accreting compact objects can be used as probes to investigate the poorly known properties of the stellar winds of hot subdwarfs.

\section{A MASSIVE WHITE DWARF WITH A SDO COMPANION}

The peculiar properties of the bright blue star HD $49798(\mathrm{~B}=8, \mathrm{~B}-\mathrm{V}=-0.27)$ attracted the attention of many astronomers since the sixties, when it was included in the (then small) group of early type subdwarfs [12]. Its first spectroscopic observations showed a dominance of He and $\mathrm{N}$ lines and radial velocity variations, pointing to a binary nature, that was later confirmed with the discovery of the orbital period of 1.5477 days [25]. In the following years several studies concentrated on a detailed modeling of the star's atmosphere. HD 49798 was classified as a subdwarf of spectral type O6, with effective temperature $\mathrm{T}_{\text {eff }}=47,500 \mathrm{~K}$, and surface gravity $\log g=4.25 \pm 0.2$ [15]. The overabundance of He and $\mathrm{N}$, and the low abundances of $\mathrm{C}$ and $\mathrm{O}$, indicated that HD 49798 is the stripped core of an initially

\footnotetext{
ae-mail: sandro@iasf-milano.inaf .it
}

This is an Open Access article distributed under the terms of the Creative Commons Attribution License 2.0, which permits unrestricted use, distribution, and reproduction in any medium, provided the original work is properly cited. 


\section{EPJ Web of Conferences}

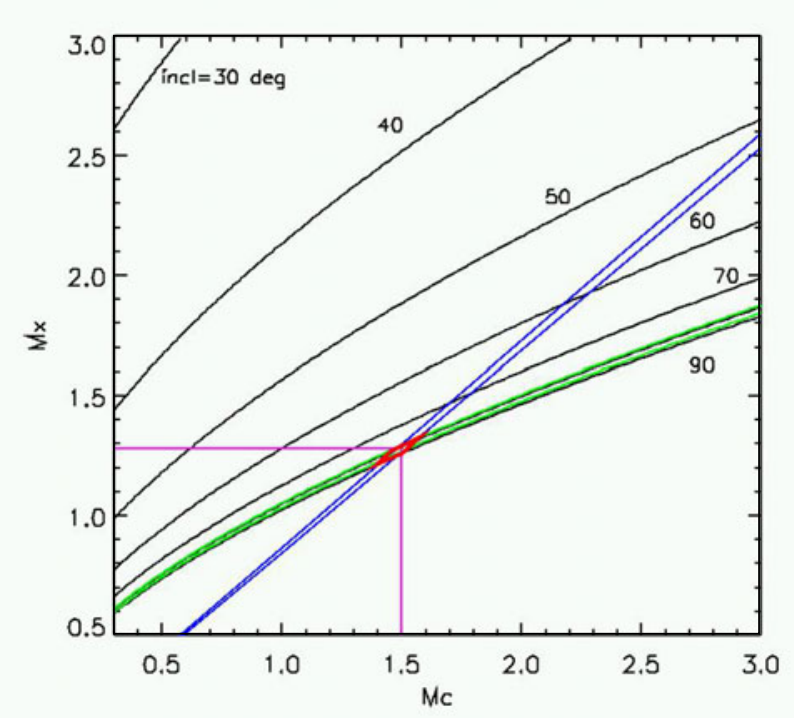

Figure 1. Masses of HD 49798 ( $X$ axis) and of its white dwarf companion ( $Y$ axis) in units of solar masses. The curved lines give, for different inclination angles, the constraints from the optical mass function. The straight lines indicate the mass ratio interval derived combining the X-ray and optical mass function. The eclipse duration constrains the inclination in the range $79-84^{\circ}$.

much more massive and larger star. The optical mass function could be measured with great precision ( $\mathrm{f}_{O P T}=0.263 \pm 0.004 M_{\odot},[24]$ ), but all the attempts to reveal the companion star, outshined in the optical/UV by the very luminous $\mathrm{sdO}\left(L \sim 10^{4} L_{\odot}\right)$, were unsuccessful.

The nature of the "invisible" companion of HD 49798 was partially clarified only in 1996, thanks to the ROSAT discovery of soft X-rays showing periodic pulsations at $13 \mathrm{~s}$ [11]. This clearly pointed to the presence of a collapsed object. However, with the poorly constrained spectrum obtained with ROSAT, it was impossible to distinguish between a neutron star or a white dwarf. In fact, depending on the spectral assumptions, the derived X-ray luminosity resulted in the range from $\sim 10^{32} \mathrm{erg} \mathrm{s}^{-1}$ up to $\gtrsim 10^{35} \mathrm{erg} \mathrm{s}^{-1}$, compatible with both possibilities.

To further advance in the understanding of this system we had to await for a long XMM-Newton pointing carried out in 2008. We strategically scheduled this observation at the orbital phase of the expected X-ray eclipse, that was never covered in previous X-ray observations. Our main objectives were to exploit the regular X-ray periodicity, which makes this system equivalent to a double spectroscopic binary, to constrain the masses of the two stars, and to get a better estimate of the source spectral parameters and luminosity. The measurement of the X-ray pulse delays induced by the orbital motion, together with the discovery of an X-ray eclipse lasting $\sim 1.3$ hours, allowed us to derive the $\mathrm{X}$-ray mass function as well as the system's inclination. This information, coupled to the already known optical mass function, gives the masses of the two stars: $\mathrm{M}_{s d}=1.50 \pm 0.05 M_{\odot}$ for the subdwarf and $\mathrm{M}_{W D}=1.28 \pm 0.05 M_{\odot}$ for its companion (Fig. 1). Furthermore, the high quality spectrum obtained with the XMM-Newton instrument showed that the total luminosity is only $\sim 10^{32} \mathrm{erg} \mathrm{s}^{-1}$, much smaller than that expected from a neutron star accreting in the stellar wind of HD 49798 . We thus concluded that this system most likely contains one of the most massive white dwarfs with a dynamical mass measurement, which is also the one with the shortest spin period $(\mathrm{P}=13 \mathrm{~s})$ [19].

The radius of HD $49798\left(1.45 \pm 0.25 R_{\odot}\right)$ is much smaller than that of its Roche-lobe, so accretion onto the white dwarf must occur through stellar wind capture. In fact, HD 49798 is one of the few O-type subdwarfs for which evidence for a relatively strong stellar wind has been obtained from 


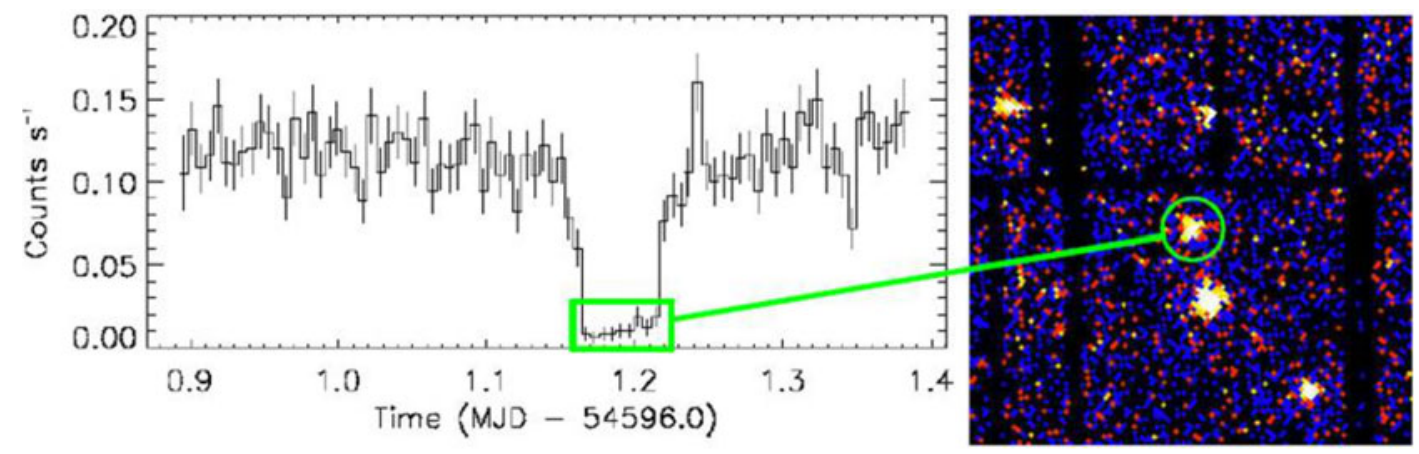

Figure 2. X-ray light curve of the May 2008 observation of HD 49798 . An eclipse lasting 1.3 hours is clearly visible. The image accumulated during the eclipse time interval shows the presence of significant emission in the $0.2-10 \mathrm{keV}$ band.

optical/UV spectroscopy. Modelling of its P-Cygni line profiles yields a mass loss rate of $\sim 3 \times$ $10^{-9} M_{\odot} \mathrm{yr}^{-1}[8]$.

The X-ray emission observed in the HD 49798 binary consists of a very soft and strongly pulsed blackbody-like component $(\mathrm{kT}=40 \mathrm{eV})$, plus a harder spectral component that dominates above $\sim 1 \mathrm{keV}$. The latter can be fit equally well by a power law with photon index $\Gamma=1.6$ or by a thermal bremsstrahlung with $\mathrm{kT}=8 \mathrm{keV}$, and shows a double peaked pulse profile [21]. Such characteristics are quite similar to those of cataclysmic variables of the polar and intermediate polar class, despite these systems are very different for what concerns their mass-donor stars and accretion geometry.

At the end of the current He-burning phase, HD 49798 will expand again and transfer He-rich matter through Roche-lobe overflow during a semi-detached phase [10], but the fraction of mass that is retained on the white dwarf is rather uncertain. Recent computations, performed assuming the mass accumulation efficiency that takes into account the wind mass loss triggered by the He-shell flashes [14], indicate that a mass of $1.4 M_{\odot}$ can be reached after only a few $10^{4}$ years [28]. However, there are other critical factors which influence the final fate of the white dwarf, such as, e.g., its composition and rotational velocity.

If HD 49798 hosts a CO white dwarf, it could be the progenitor of an over-luminous type Ia supernova, since the fast rotation can increase the mass stability limit above the value for non-rotating stars. Massive white dwarfs are expected to have an ONe composition, but again the high spin might play a role here, since it can lead to the formation of $\mathrm{CO}$ white dwarfs even for high masses. The fact that this system originated from a pair of relatively massive stars $\left(\sim 8-9 \quad M_{\odot}\right)$ might suggest that it could be the progenitor of a type Ia supernova with a short delay time. However, the delay time might be considerably longer if the explosion has to await that the white dwarf spins down [2].

Alternatively, if the companion of HD 49798 is an ONe white dwarf, an accretion-induced collapse might occur, leading to the formation of a neutron star. The high spin rate and low magnetic field make this white dwarf an ideal progenitor of a millisecond pulsar. The evolution of systems like this one could be a promising scenario for the direct formation of millisecond pulsars, i.e. one not involving the recycling of old pulsars in accreting low-mass X-ray binaries.

Besides their obvious interest in the context of the binary evolutionary modes, these X-ray observations are interesting for the study of the sdO star itself. It is remarkable that X-rays have been detected also when the white dwarf is eclipsed by the much larger sdO companion (Fig. 2). The observed emission, with a luminosity of $\sim 2 \times 10^{30} \mathrm{erg} \mathrm{s}^{-1}$, could be the first detection of a sdO star in the X-ray band [21]. The observed ratio of X-ray to bolometric luminosity of a few $10^{-7}$ is in accordance to that of main sequence and supergiant $\mathrm{OB}$ stars. Alternatively, the X-rays seen during the eclipse could be due to the reprocessing of the white dwarf emission in the stellar wind. Our most recent XMM-Newton 

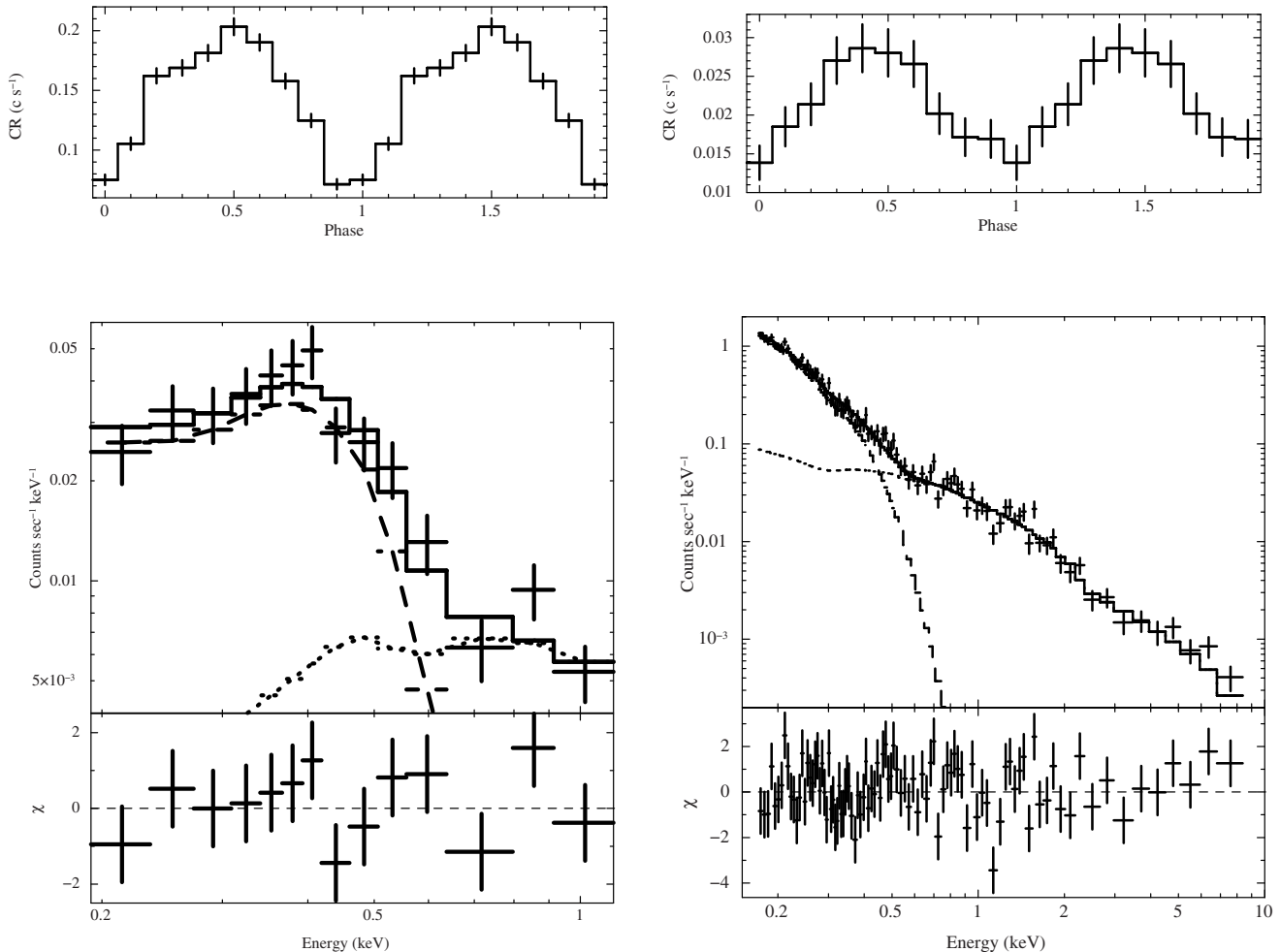

Figure 3. Comparison of the X-ray light curves and spectra of HD 49798 (left) and BD $+37^{\circ} 442$ (right). The top panels show the light curves in the range $0.2-0.5 \mathrm{keV}$ folded at the periods of $13.2 \mathrm{~s}$ and $19.2 \mathrm{~s}$, respectively for $\mathrm{HD} 49798$ and $\mathrm{BD}+37^{\circ} 442$. The bottom panels show the spectra with the best fit blackbody plus power-law models. Note that the flux of $\mathrm{BD}+37^{\circ} 442$ is much lower than that of HD 49798 . Hence its spectral parameters are not well constrained. This implies a large uncertainty on its total X-ray luminosity.

observations showing the presence of emission lines of $\mathrm{N}$ and $\mathrm{Ne}$ in the eclipse spectrum seem to favor this interpretation.

\section{AN EXTREME HE STAR WITH A NEUTRON STAR OR WHITE DWARF COMPANION}

Prompted by the possible detection of X-ray emission from HD 49798 itself during the white dwarf eclipse, we requested XMM-Newton observations of a supposedly single hot subdwarf. This led to the discovery of soft X-rays with a periodicity of $19.2 \mathrm{~s}$ from the luminous sdO BD $+37^{\circ} 442$, indicating also in this case the presence of a compact object [17]. This was quite surprising because all the data reported in the literature for $\mathrm{BD}+37^{\circ} 442$ did not show any evidence of a binary companion: infrared observations did not show any excess emission [26], and no signatures of a binary nature were seen in spectroscopic or photometric data $[4,5,16]$. On the other hand, the few radial velocity measurements found in the literature give inconsistent values $\left(\mathrm{V}_{r}=-156.4 \pm 1.1 \mathrm{~km} \mathrm{~s}^{-1}[23], \mathrm{V}_{r}=-94 \pm 1 \mathrm{~km} \mathrm{~s}^{-1}\right.$ [3]), which could be caused by binary motion. Further spectroscopic observations are clearly needed to search for an orbital period in $\mathrm{BD}+37^{\circ} 442$.

The X-ray emission from $\mathrm{BD}+37^{\circ} 442$ has a soft spectrum well described by a blackbody with temperature $\sim 45 \mathrm{eV}$ plus a weak power-law component. This is very similar to the spectrum of HD 49798 (Fig. 3). The best fit X-ray luminosity is $\sim 10^{33} \mathrm{erg} \mathrm{s}^{-1}$ (for a distance of $2 \mathrm{kpc}$, [1]), but, 
Ageing Low Mass Stars: From Red Giants to White Dwarfs

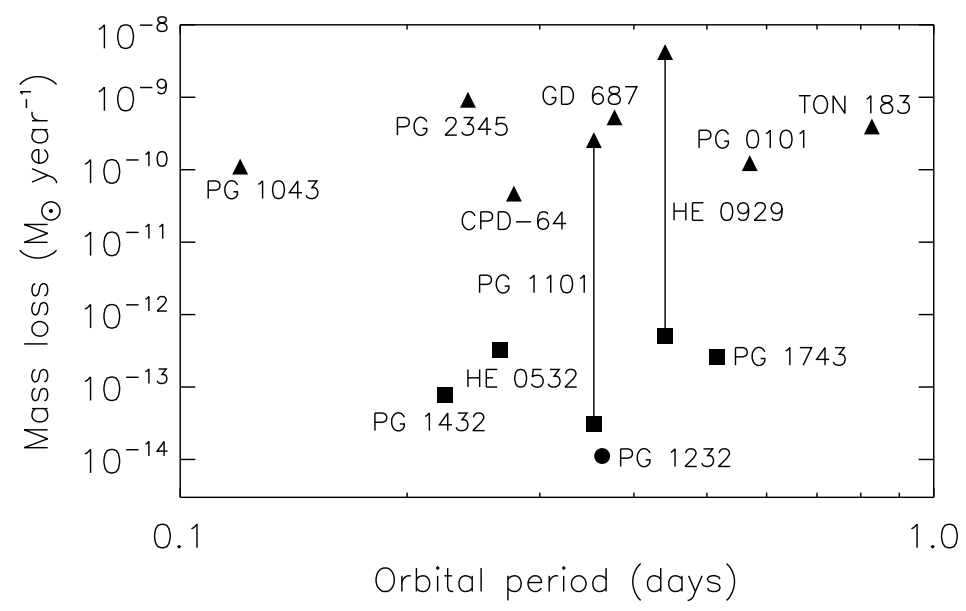

Figure 4. Upper limits $(3 \sigma)$ on the $\mathrm{sdB}$ mass loss rates in the case of a white dwarf (triangles), a neutron star (squares), or a black hole (circle) companion.

due to the uncertainties in the X-ray blackbody temperature, the bolometric X-ray luminosity could be between $\sim 10^{32}$ and $\sim 10^{35} \mathrm{erg} \mathrm{s}^{-1}$.

The observed pulsations clearly indicate the presence of a white dwarf or a neutron star, most likely powered by accretion from the stellar wind of $\mathrm{BD}+37^{\circ} 442$, which is loosing mass at a rate of $\sim$ $3 \times 10^{-9} M_{\odot} \mathrm{yr}^{-1}$ and with a wind terminal velocity of $\mathrm{v}_{\infty}=2,000 \mathrm{~km} \mathrm{~s}^{-1}$ [13]. Simple computations, assuming a canonical velocity law for the wind and Bondi-Hoyle accretion, show that a neutron star orbiting $\mathrm{BD}+37^{\circ} 442$ with a period of a few days would accrete from the wind at a sufficiently high rate to produce the observed X-rays. Alternatively, the accreting companion could be a white dwarf, but this would require a larger accretion rate that would only be possible if the accretion is due to Roche lobe overflow.

\section{SDB WITH CANDIDATE COMPACT COMPANIONS}

We observed with the Swift satellite a few sdB binaries selected from the MUCHFUSS project, which aims at finding hot subdwarfs with compact companions by means of radial and rotational velocity measurements in the optical band [7]. None of the observed targets was detected in the X-ray band, and we could set upper limits on their luminosity in the range $\mathrm{L}_{X} \sim 10^{30}-10^{31} \mathrm{erg} \mathrm{s}^{-1}$ [20]. Although this negative result does not allow us to confirm the presence of compact stars inferred from the optical data, the upper limits on $\mathrm{L}_{X}$ can be used to constrain the poorly known properties of the sdB stellar winds ${ }^{1}$.

The luminosity upper limits can be converted into limits on the mass loss rates $\dot{M}_{\mathrm{W}}$ from the sdB stars, assuming for simplicity Bondi-Hoyle accretion from the stellar wind onto the compact objects. This is justified by the fact that in these systems the sdB stars do not fill their Roche lobes. Our assumptions lead to conservative upper limits on $\dot{M}_{\mathrm{W}}$, since most wind-accreting neutron stars in highmass X-ray binaries show an X-ray luminosity larger than that predicted by the simple Bondi-Hoyle theory.

The resulting limits on $\dot{M}_{\mathrm{W}}$ are plotted in Fig. 4, where different symbols are used to indicate the assumed compact object. For the systems likely hosting white dwarfs (triangles) the limits are above

\footnotetext{
${ }^{1}$ We assume that compact objects are really present in these systems. An alternative trivial explanation for their non-detection is that some of the assumptions made to infer the presence of compact objects is wrong. However this is rather unlikely [6] and we will not consider further this possibility.
} 
$\dot{M}_{\mathrm{W}} \sim 5 \times 10^{-11} M_{\odot} \mathrm{yr}^{-1}$. Although they are not particularly constraining for the sdB wind properties, we note that they represent one of the few observational results in this field. Deeper X-ray observations of the closest candidates (e.g. PG 0101+039 and CPD -64 481) with more sensitive satellites like XMMNewton or Chandra should be able to detect accreting white dwarfs, if their sdB companions lose mass at a rate $\dot{M}_{\mathrm{W}} \sim 10^{-12}-10^{-11} M_{\odot} \mathrm{yr}^{-1}$, as predicted by theoretical wind models.

More interesting constraints can be inferred from the binaries likely containing neutron stars or black holes (PG 1432+159, HE 0532-4503, PG 1232-136, and PG 1743+477). The lack of X-ray emission implies that the sdB stars in these systems have rather weak winds, with $\dot{M}_{\mathrm{W}}<3 \times 10^{-13} M_{\odot} \mathrm{yr}^{-1}$, which is significantly below the predictions of theoretical models [27], if a solar metallicity is assumed. A metallicity $Z=0.3 Z_{\odot}$, or lower, is required for these sdB stars to be consistent with the derived upper limits.

We acknowledge financial contribution from the agreement ASI-INAF I/032 /10/0.

\section{References}

[1] Bauer, F. \& Husfeld, D. 1995, A\&A 300, 481

[2] Di Stefano, R et al. 2011, ApJ 738, L1

[3] Drilling, J.S. \& Heber, U. 1987, in IAU Colloq. 95: Second Conference on Faint Blue Stars, ed. A.G.D. Philip, D.S. Hayes, \& J.W. Liebert, 603-606

[4] Dworetsky, M.M., Whitelock, P.A., \& Carnochan, D.J. 1982, MNRAS 201, 901

[5] Faÿ, T., Honeycutt, R.K., \& Warren, Jr., W.H. 1973, AJ 78, 246

[6] Geier, S., Heber, U., Podsiadlowski, P., et al. 2010, A\&A 519, A25

[7] Geier, S. 2011, A\&A 530, 28

[8] Hamann, W. 2010, Astroph. Sp. Science 119, 1

[9] Han, Z., Podsiadlowski, P., Maxted, P.F.L., Marsh, T.R., \& Ivanova, N. 2002, MNRAS 336, 449

[10] Iben, I.J. \& Tutukov, A.V. 1994, ApJ 431, 264

[11] Israel, G.L., Stella, L., Angelini, L., et al. 1997, ApJ 474, L53

[12] Jaschek, M. \& Jaschek, C. 1963, PASP 75, 365

[13] Jeffery, C.S. \& Hamann, W.-R. 2010, MNRAS 404, 1698

[14] Kato, M. \& Hachisu, I. 2004, ApJ 613, L129

[15] Kudritzki, R.P. \& Simon, K.P. 1978, A\&A 70, 653

[16] Landolt, A.U. 1973, PASP 85, 661

[17] La Palombara, N. et al. 2012, ApJ 750, L34

[18] Maxted, P.F.L., Heber, U., Marsh, T.R., \& North, R.C. 2001, MNRAS 326, 1391

[19] Mereghetti, S., Tiengo, A., Esposito, P., et al. 2009, Science 325, 1222

[20] Mereghetti, S., Campana, S., Esposito, P., La Palombara, N., \& Tiengo, A. 2011a, A\&A 536, A69

[21] Mereghetti, S., La Palombara, N., Tiengo, A., et al. 2011b, ApJ 737, 51

[22] Morales-Rueda, L. et al. 2003, MNRAS 338, 752

[23] Rebeirot, E. 1966, C.r.hebd. Seanc. Acad. Sci. Paris 262, 1105

[24] Stickland, D.J. \& Lloyd, C. 1994, The Observatory 114, 41

[25] Thackeray, A.D. 1970, MNRAS 150, 215

[26] Thejll, P., Ulla, A., \& MacDonald, J. 1995, A\&A 303, 773

[27] Vink, J.S. \& Cassisi, S. 2002, A\&A 392, 553

[28] Wang, B. \& Han, Z. 2010, Research in Astronomy and Astrophysics 10, 681 